\title{
The Theoretical didactic approach to the counterexample in mathematics
}

\author{
Armando Morales-Carballo ${ }^{1}$, Edgardo Locia-Espinoza ${ }^{1}$, Melvis Ramírez-Barragán ${ }^{1}$ \\ José María Sigarreta Almira ${ }^{1}$, Otilio B. Mederos ${ }^{2}$ \\ armando280@hotmail.com, lociae999@htmail.com, rbmelvis1789@gmail.com, \\ josemariasigarretaalmira@hotmail.com, omederosa@gmail.com \\ ${ }^{1}$ Universidad Autónoma de Guerrero, México. \\ ${ }^{2}$ Universidad Autónoma de Coahuila, México.
}

\begin{abstract}
This article describes a theoretical-didactic approach to the counterexample within mathematics and its process of teaching-learning, emphasizing the importance of inducing a logical thinking by introducing counterexamples as a process of maturation of mathematical thinking. In addition, it is argued that the counterexamples are not very used in the teaching of mathematics, unlike the important role they have in the professional mathematic activity.
\end{abstract}

Keywords:Counterexample, mathematics, validation, teaching, learning.

Subject Classification:Mathematics Subject Classification.

Type (Approach): Literary Analysis.

\section{Introduction}

The papers reported by (Arnal-Bailera\&Oller-Marcén, 2012, Lee, 2016, Lozano, 2015, Stylianou, Chae, \& Blanton 2006) agreed that one of the main problems where students and professors have difficulties for demonstration in mathematics, are the difficulties about argumentation and proof, and this influences in the comprehension of concepts and their definitions, properties and logical relations between parts that structure the axiomatic-math.

In particular, the relative researches to the demonstration in the teaching of mathematics reported by (Hersh, 1993, Antibi, 1998; Mitchel, 1996) argue that while is true that in mathematics the concept of demonstration is fundamental, there are a lot of professors who cannot understand why mathematicians give to the demonstration a privileged status, others do not identify between empirical arguments and deductive arguments; in particular, it has been identified that the professors have difficulties to apply correctly definitions, theorems and formulas, professors get confused between sufficient conditions and necessary conditions, they use uncertain conclusions that are frequently false.

In the field of mathematics, by assuming the law of the excluded third, two tools are enabled to prove the truth of an affirmation: it is proved and with this it gets established that it is true, or a counterexample is exhibited to refute it. However, in the teaching practice, we have identified in both professors and students the existence of difficulties in the utility of this law as a resource of demonstration, and it can be seen, the frequent non-use of the counterexample as a tool that makes possible the refutation of affirmations. Although the systematic use of counterexamples to refute affirmations is not present in the teaching process, the papers reported by (Morales, 2008 y Locia, 2000) establish that the good comprehension about validation 
mechanisms must necessarily go through a careful analysis of the functioning of refutations, in general, and of the use of counterexamples, in particular.

From the purely mathematical point of view, the counterexample has a well-defined status. When this notion is approached from the field of logic, a closed logical statement of the type $\forall x_{s} p(x)$ is proposed. To prove its invalidity, it has to be proved by the law of the excluded third that the statement $\neg(\forall \mathrm{x}, \mathrm{p}(\mathrm{x}))$ is true, in other words, an $\mathrm{x}_{0}$ must be produced, such as $\neg \mathrm{p}\left(\mathrm{x}_{0}\right)$. Thus, the rule of the counterexample is enunciated in the following way: To prove that a statement of universal character is false, it is sufficient to show a counterexample (Arsac, 1997).

With this background, this paper inquired about the didactic conception of the counterexample in a group of fifteen professors in middle school, pre-university and university, and in training. In particular, the following conceptions were explored:

1. Professors recognize counterexamples only when they are fully and normally present in the standard conditions we have identified: At length: a closed statement of type $\forall \mathrm{x}, \mathrm{p}(\mathrm{x})$ is proposed, the students must to declare its invalidity $\neg(\forall \mathrm{x}, \mathrm{p}(\mathrm{x}))$, make a $\mathrm{x}_{0}$ such as $\neg \mathrm{p}\left(\mathrm{x}_{0}\right)$ and to indicate that this prove that.

2. Professors recognize counterexamples in larger, less rigorous and incomplete situations, which will prove a different didactic conception of the logical conception.

\section{Theoretical-didactic approach to the counterexample.}

\subsection{Identification of the counterexample object.}

Considering the definition given by Kleene:

"A formula $\mathrm{F}$ of the predicate calculus is not exactly valid if $\mathrm{F}$ is falsifiable in the following sense: $\mathrm{A}$ non-empty domain $D$ exists and an assignment in $D$ of the parameters of $F$ that give the value (false). That assignment will be called counterfeit for $F$ in $D$ and $F$ will be called falsifiable in this $D$ [...]

The system formed by this $\mathrm{D}$ and this assignment may be appointed as constituting a counterexample to $\mathrm{F}$.

Replacing $\mathrm{F}$ for $\mathrm{T}$ (true) we obtain the notions of satisfiable of assignment that satisfies. [...] and of example" (Kleene, 1967, pp 284).

1. It is immediately observed that the term "counterexample" is not defined as a term of the predicate calculus, and not even of the model theory of the predicate calculus. It is a metalinguistic term, intermediary between the language of the constructor and the constructed language. It remits to a certain organization of a set of formulas of predicate calculus constituted in element of "demonstration".

2. In this definition is said that a counterexample is a pair $(D, \delta)$ formed by a domain $D$ and of an assignment $\delta$ (in other words an $n$-tuple of values of the parameters of $F$ ), but in fact for this pair to be an example or a counterexample, it is necessary to remit it to an argument $F$, as a result $(D, \delta, F)$ form a counterexample whether $\delta \in \mathrm{D}$ and whether $\delta(\mathrm{F})$ takes the value $\mathrm{f}$ and an example whether $\delta \in \mathrm{D}$ and whether $\delta(\mathrm{F})$ takes the value $t$. The everyday formulation in mathematics becomes in " $\delta$ is a counterexample of $F$ in the domain $\mathrm{D}$ ". In this case, the use emphasizes the assignment $\delta$, which becomes "the counterexample", while the other two terms are relegated to the second plane as conditions or even they merge in the context.

3. In logic, the notion of the counterexample only appears in model theory. There the validity of formulas are examined by the assignments of their variables to different domains. In the theory of demonstration this notion does not appear. What could correspond to it would be the production of two contradictory closed statements such as $\forall \mathrm{x}, \mathrm{p}(\mathrm{x})$ and $\neg(\forall \mathrm{x}, \mathrm{p}(\mathrm{x}))$, in other words $\exists \mathrm{x}, \neg \mathrm{p}(\mathrm{x})$. But $X$ is not "displayed" because there is not domain of realization to consider and the vocabulary of the "examples" do not have object. 
However, in mathematical practice, especially at the elemental level, the languages and the methods of two theories are used simultaneously (the concept of valid consequence gets confused with the deduction, for example).

4. In the definition of Kleene, only the formulas $F$ that are not formally closed may be the object of a counterexample, inasmuch as they have to possess at least an assignment $\delta$ in at least a domain D. Notwithstanding the counterexample, in fact, is going to use it to refute the validity of closed formulas. In other words, whether an any closed formula $P$ is given of predicate calculus and a domain $D$. This domain determines the possible assignments of some parameters of $P$. We consider the predicate $F$ (we should say $F(P)$ ) obtained by suppressing in $P$ the quantifiers relating to those parameters. In this $F$ which is susceptible of receiving examples and/or counterexamples in $D$.

\subsection{Counterexamples in the construction of mathematical knowledge}

\subsubsection{Heuristics of mathematical discovery. Polya's work.}

In his studies about heuristics of mathematical discovery, G. Polya works with the topic about strategies to get new knowledge (Polya, 1958). He affirms that although is true that finished mathematics, presented in a definitive form, they seem purely demonstrative by involving only theorems and demonstrations, the same is not true for mathematics in gestation. In the construction of new mathematical knowledge, is necessary combine the observations and rely on analogies. It is necessary to try and try to get conjectures and guess their demonstrations. Poly analyses, from concrete examples, search strategies based on the processes of induction, analogy, generalization and particularization and he says that such processes are particular cases of a type of reasoning very used in mathematics: plausible reasoning. The scheme of such reasoning is the following: a conjecture $A$ is reached (which is believed to be true) and from $A$ is deduced an affirmation $B$. If $B$ is false, $\mathrm{A}$ becomes more plausible.

Although Polya's works are not focused on the use of counterexamples in mathematics, what it is important to underline is the proposed procedure, based on the interactions between train and error to surmise and proof. The detection of an error, a contradiction or an omission is well received as an important step in the construction of the proof.

\subsubsection{The logic of the mathematical discovering. Lakatos' work.}

The book I. Lakatos (Lakatos, 1976) is a significant presentation of the operation of the counterexample in the logic of mathematical discovering. The author presents the methodology of mathematical discovering through the logic of proofs and refutations, through a fictitious debate between a professor and their students in a class where the Euler's conjecture is discussed. This conjecture aims to establish a relation between the number of faces, number of edges and number of vertexes of a polyhedron. It is convenient to mention that the stages of this debate are real: They are all those through which mathematicians passed in the search for a definitive proof of Euler's theorem.

The Euler conjecture and a proposal of a proof. The analysis carried out by Lakatos on the logic of the mathematical discovering starts with the search of a relation between the number $V$ of vertexes, the number $A$ of edges and the number $C$ of faces of a polyhedron, similar to that between the number $V$ vertexes and the number $\mathrm{A}$ of sides of a polygon, that is $\mathrm{V}=\mathrm{A}$. After several trials and errors, one of the participants in the debate presents the Euler conjecture to the group: In every polyhedron $\mathrm{V}-\mathrm{A}+\mathrm{C}=2$.

In the absence of a definitive proof, Lakatos shows how he can presents a conjecture what he calls "a mental experiment" or "quasi-experiment", it is characterized mainly for the decomposition of the primitive conjecture into sub-conjecture or lemmas that open up new instances of criticism and contrast. Thus, the mental experiment to which the Euler conjecture is subjected consists of the following lemmas or sub-conjectures (this proof is inspired by that given by Cauchy in 1813): 
1. A polyhedron is flattened by removing one of their faces. So that establish $\mathrm{V}-\mathrm{A}+\mathrm{C}=1$ for a flat graph.

2. The flat graph is triangulated by drawing diagonals on faces that are not triangles. In this operation is added one face and one edge are added at each step, therefore $\mathrm{V}-\mathrm{A}+\mathrm{C}$ keeps constant.

3. The triangles are eliminated, one by one, with one of the following two operations: Delete one side or delete two sides and one vertex. Thus, if $\mathrm{V}-\mathrm{A}+\mathrm{C}=1$ before one of the two operations, then $\mathrm{V}-\mathrm{A}+\mathrm{C}=1$ after. At the end of this procedure, there keeps only one triangle for which $\mathrm{V}-\mathrm{A}+\mathrm{C}=1$, therefore it can pretend that the conjecture is proven.

Counterexamples and dialectic of proof and refutation. From this proof, Lakatos illustrates the operation of mathematics from the formulation of conjectures, to the confirmation or refutation of them. He tells how the examples appear that do not fit with the conjecture or with the proof (counterexamples), showing their function of falsification or refutation. The criterion of rigor that Lakatos gives is that, if any counterexample comes to refute the conjecture or its proof, this (the conjecture) must be accepted as true. He calls global counterexample to that counterexample that refutes the conjecture and local counterexample to the one that refutes its proof (or one of its lemmas). A local counterexample has the characteristics that make the proof not valid for that case, however it verifies the conjectured proposition. These refute one of the lemmas, without refute the conjecture; they criticize the proof because in that example, the property was supposed to be valid is not fulfilled. What is refuted is an implicit lemma and therefore, the proof. On the other hand, the presence of global counterexamples of the theorem produces a conflict between the concept, the conjecture and its proof. This conflict involves the conjecture or the proof, and it can be solved in different ways, including adjusting the definition of the concept or determining the abandonment of the conjecture. A global counterexample can be at the same time local, in other words, to refute a lemma (or sub-conjecture) of the proof: it can only refutes the conjecture, in other words, it is global but not local. This case is treated in a special way by Lakatos. All this allows to establish different working methods with the objective of the validity. According to these methods, the counterexamples can lead respectively to try to revise the conjecture, the involved terms or its proof. Method of surrender, of exclusion of monsters, of adjustment of monsters, of exclusion of exceptions, and of incorporation of lemmas. Lakatos shows how the debate about incomplete demonstrations is one of the preponderant elements of progress in mathematical discovery. Especially when the putative demonstrations imply implicit, they resort to evidence ("hidden lemmas"), they use notions that are not totally defined, where errors and contradictions can be dissimulated. It is precisely the existence of these errors and these contradictions that is productive. The fact of overcoming them becomes a source of progress. The presentation of a counterexample to expose a contradiction can lead to questioning about the proof looking for the "hidden lemmas" that give rise to the counterexample. Thus, in the interaction of the proof and the counterexample, the latter not only serves to expose the error, but it also leads to retake ideas of implicitly defined objects (which in view of the necessities of its use we will be obliged to specify little little to explain their definitions), and to realize the different meanings of the terms used to express the demonstrations.

\subsubsection{The role of counterexamples in the production of mathematical knowledge.}

Lakatos points out that counterexamples have a much more important role in the search for results in mathematics than what makes us believe their place in the classical (axiomatic) presentation of these same results. It was identified, how, in his project to oppose the characteristics of mathematics made with mathematical activity, the counterexample has the role of a revealing. It allow him to show that dialectic of research is not well represented by the order and by means of classical exposition and demonstration.

More precisely, Lakatos shows the driving role of the attempts at proof and refutations in the elaboration of a mathematical theory and the role of counterexamples in this dialectic. His modelling of the debate of Euler 
conjecture allows to understand well the different uses of the counterexample as a means of control of the validity of conjectures, demonstrations, conditions of validity, as a means of choosing definitions and so on, and this modelling also shows how, progressively, the process of mathematization hides and eliminates these counterexamples by: relegation of exceptions, incorporation of lemmas and so on.

Once the construction work is finished, the scaffolding of counterexamples disappears from the text; this can only be restored-at least in part- by the reader, as an activity of interrogation and comprehension of the text. This restoration is obviously very partial.

\subsubsection{Use of the counterexample in the study of content and extension of the concepts.}

A concept is a generalized mental model of two classes, a class of certain features (attributes, properties) or relations between grouped objects and the other class constituted by objects with that characteristics. They are called essential properties of a concept to characteristics of objects modeled in it, each of which is necessary to distinguish the objects that correspond to the concept of others and all in sufficient sets for this purpose. The concept of object is taken as primary; namely, without definition.

Every concept always has two logical characteristics: content and extension. A set of essential properties of modeled objects in the concept, which is sufficient to distinguish the new objects of the concept, constitutes its content. Content is an indispensable factor of any concept, so it cannot exist a concept lacking content, in which consequently no property is conceived. The extension of a concept is the kind (set) of models of objects that this concept covers. Extension is a logical characteristic of the concept as indispensable as its content. The model of the object is understood as the collection of its registers of representations (graphic, analytical and so on).

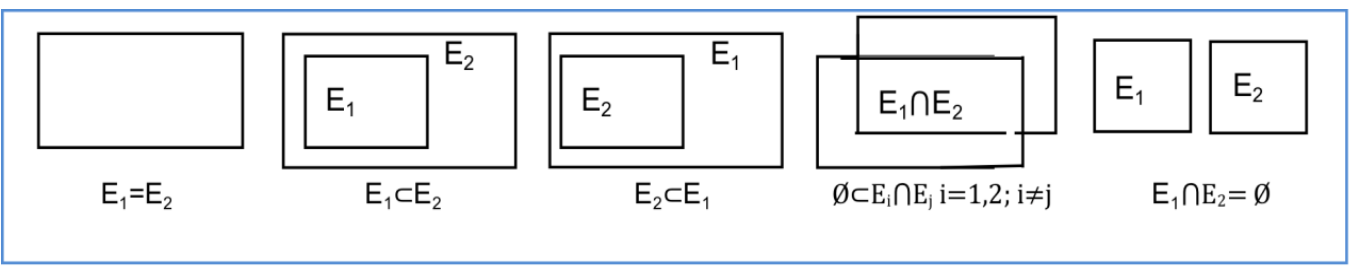

Image 1: The five possible relations between extensions $E_{1}$ and $E_{2}$, two concepts.

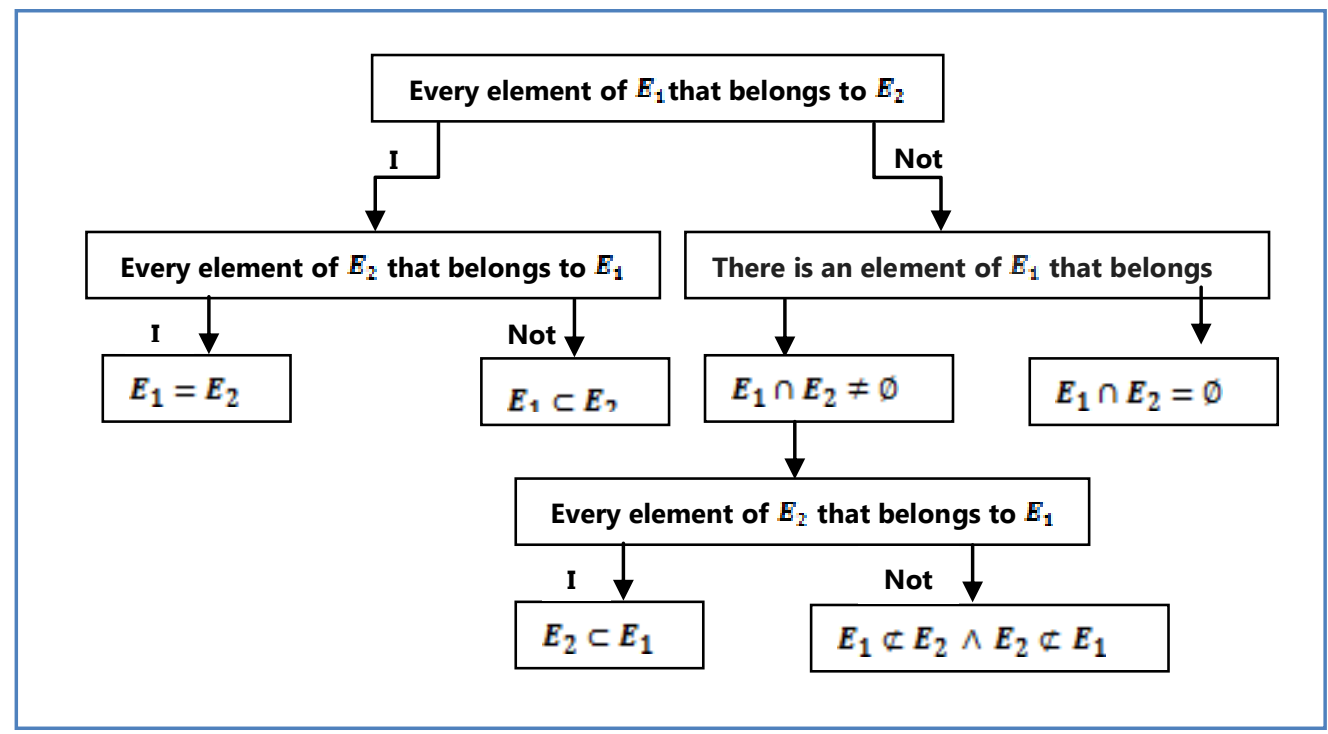

Image. 2: Conjunctist relation of the four extensions of $E_{1}$ and $E_{2}$. 
In this work a concept is indicated by the pair $\left(\square_{1}\right)$ or simply by $E \square$ when there are not doubts; where $E \square$ denotes to the extension of concept and for $C \square$ to its content. Different collections $C$ of properties that only the elements of $\square E$ can be found. It is usual to indicate the content by the collection of properties $\left\{P_{i}\right\} i \in I \square$ that has been chosen, where $I \square$ is a set of indexes. That indicates the objects that belong to the extension of the concept simultaneously fulfill all those properties, or what is the same, fulfill the unique property $A i \in I P_{i}$. Extensions $\square E 1$ and $\square E 2$ of two concepts $(\square E 1,1)$ and $(E 2,2)$ fulfill only one of the five relations that are shown in the image 1.

To determine the conjunctist relation that the extensions $\square E 1$ and $\mathrm{DE} 2$ have, it is necessary demonstrate, or refute, one or several universal $\mathrm{P}$ propositions of the form "All element of the class $\square E 1$ (E2 respectively) belongs to the class $\square E 2$ (E1 respectively)" and existential of the type "There is an element of the class $\square E 1$ ( $\square E 2$ respectively) that belongs to the class $\square E 2$ ( $\square E 1$ respectively)", image 2 .

To refute a proposition of this kind it is sufficient to find, or construct, a counterexample. To refute the proposition "All continuous function of real variable $f: D \rightarrow R$ is differentiable in all its domain $D \square$ ", it is sufficient to construct an element of extension $C(D)$ of concept of continuous function on $D$ that does not belong to the extension $D(D)$ of concept of differentiable function on $D$. This proposition is equivalent to the preposition "There is a continuous function that is not differentiable at a point of its domain".

\subsubsection{The role of counterexamples in didactic of mathematics}

In the development of mathematics propositions that correspond to implications are used. Examples and counterexamples are very useful tools to show, respectively, the existence of objects that fulfil all the hypotheses and that none of these can be eliminated. In this direction we agree with Tsamir, Tirosh and Levenson (2008), when in the scope of general principles of the formation of concepts, the particular cases of a concept can be called examples.

In the statement of the Mean Value Theorem are imposed on a real function $f$ with domain $[a, b], a<b$, the two following conditions, $f$ is continuous on $[a, b]$ and $f$ is derivable on $(a, b)$.

Before studying the Rolle and Mean Value Theorems, many students have not worked with elements of collection $C[a, b] \cap \mathrm{D}(a, b)$ for which there are not derivables of the function in $\square a$ and $b$.

The function $f$ defined by $(x)=\sqrt{1-x^{2}}$ belongs to the class $C[-1,1] \cap D(-1,1)$ and there are not derivables $f^{\prime}(-1)$ and $f^{\prime}(1)$. The real function $f$ with domain $[-1,1]$ defined by $f(x)=(1 / 6) x^{2}$ if $x<0$ y $f(x)=x$ if $x \geq 0$, is continuous on $[-1,1]$, but does not comply with the property of derivability over the entire interval, since it is only derivable over $(-1,1) \backslash\{0\}, f^{\prime}(x)=(1 \backslash 3) \mathrm{x}$ if $x<0, f^{\prime}(0)$ does not exist and $f^{\prime}(\mathrm{x})=1$ if $x<0$. This function $f$ does not meet the equality $f^{\prime}(c)=[f(1)-f(-1)] / 2$ for any $c$ de $(-1,1) \backslash\{0\}$; since the equality is only met for $c=$ $15 / 12$, and in this case $c$ does not belong to $(-1,1)$.

\section{Results and Discussion}

Approach to the didactic use of the counterexample. In the field of mathematics teaching-learning, works have been developed concerning the formulation of conjectures and the use of counterexample for the treatment of concepts, properties and mathematical relations. In particular, in the investigations reported by Weber (2009), Komatsu (2010), Ko and Knuth (2013), Giannakoulias, Mastorides, Potari and Zachariades (2010), Komatsu, Jones, Ikeda and Narazaki (2017), Garcia \& Morales (2013), Klymchuk (2010), Zazkis \& Chernoff (2008), Huang (2014) have identified that they are aimed at students, professor in service and in training, and towards postgraduate students, in each of them it becomes clear that the counterexample is a didactic tool that favors the processes of validation and comprehension of mathematical knowledge.

From the analysis of the studies reported in aforementioned works, regarding the use of counterexample; we identify the following methodological implications: 
1. It allows to stimulate the students' reasoning of the how and why of the processes followed to reach conclusions, and to diminish the memory and algorithmic learning procedures: this implication was identified in reach works directed towards the student in the school levels (elementary school, middle school, high school, undergraduate and postgraduate) to deal with specific content of mathematics such as: arithmetic, algebra, geometry, calculus and analysis, number theory and so on.

2. It allows the functional structuring of the logical-mathematical reasoning: this implication was identified in the proposals related to the study of the proof and the production of the counterexample for the validation of results.

3. Encourages students to reflect on essential aspects of mathematics by highlighting the importance of the rules, principles, theorems and properties associated with mathematical objects: this implication was identified in the student's activity, the professor's and the professor in training.

4. It allows to identify through the constructive process of knowledge the basic and invariant characteristics and properties of mathematical objects: this implication was identified from counterexamples proposals that favored the refutation of conjectures about the definition of concepts of mathematical objects in concrete subjects of arithmetic, geometry, algebra, calculus, number theory, and so on.

5. Reveals misconceptions, and requires attention to each detail of the process by improving the understanding of mathematical concepts and properties: this implication was identified when conceiving the counterexample in its semantic connotation and its use as a didactic tool to favor validation processes; and in this process, the refutation through the counter-example proposal was fundamental.

In previous sections we have seen that, in mathematics, the word counterexample has a well-defined meaning and status and that its role in the construction of mathematical knowledge through the dialectic of proof and refutation is of great importance despite that, once the mathematical theories have been completed and presented in an axiomatic manner, the counterexamples disappear completely from the mathematical texts.

\section{Conclusions}

- In the professional mathematical activity the counterexamples have a very important role. However, this role is not present in the axiomatic presentation of knowledge.

- The bias thus introduced in the didactic transposition of mathematics, has consequences in teaching where counterexamples have a modest role, little representative that they have in professional mathematical activity. Thus counterexamples are methods of reasoning rather than objects of teaching.

-The theoretical-didactic elements of approach to the counterexample provide the theoretical foundation of attention to the mathematical content and give the methodological basis for the elaboration of proposals that contribute in the teaching-learning processes of mathematics, in a specific way: the treatment of concepts and their definitions, theorems and their demonstrations.

\section{References}

1. Antibi, A (1988). Etude surl'enseignement de méthodes de démonstration. Enseignement de la notion de limite: reflexions, propositions. PhD thesis, Toulouse. Universidad Paul Sabatier.

2. Antibi, A (1999). La motivación en matemáticas: ¿la del profesor? ¿la del alumno? Actas de las 9as Jornadas para el aprendizaje de las matemáticas. 22-25.

3. Arnal-Bailera, A. y Oller-Marcén, A. M. (2017). Formación del Profesorado y Demostración Matemática. EstudioExploratorio e Implicaciones. Bolema, 31, 57,135-157. 
4. Arsac, \&Mante, M (1997). Situations d'initiations au raisonnementdéductif. Educational Studies in Mathemtics, 33 (1): 247-280

5. Arsac, G (1987). L'origine de la démostration: essaid'épistemologiedidactique. Recherches en didactique des mathématiques, 8(3): 267-312.

6. Arsac, G (1988). Les recherchesactuellessurl'apprentisage de la démonstration et les phénoménes de validation en France. Recherches en didactique des mathématiques, 9(3): 247-280.

7. Brousseau, G (1986). Fondements et méthodes de la didactique des mathématiques. Recherches en didactique des mathématiques, 7(2): 33-115

8. Chazan, D (1993). High school geometry students justification for their views of empirical evidence and mathematical proof. Educational studies in mathematics, 24(4): 359-387

9. García, O. y Morales, L. (2013). El Contraejemplo como Recurso Didáctico en la Enseñanza del Cálculo. Revista Iberoamericana de Educación Matemática, 13, 161-175.

10. Gelbaum, R. \& Olmsted, J. (1964). Counterexamples in analysis. San Francisco. Holden Dav, Inc.

11. Giannakoulias, E., Mastorides, E., Potari, D., y Zachariades, T. (2010). Studing teachers' mathematical argumentation in the contexto of refuting students invalid claims. The Journal of Mathematical Behavior, 29, 160-168.

12. Huang, Ch.(2014). Engineering students' generating counterexample of calculus concepts. Global Journal of Engineering Education, 16(2), 93-97.

13. Huchecorne, B. (1988). Les contre-exemples en mathématiques. París. Ellipses.

14. Hersh, R. (1993). Proving is convincing and explaning. Educational studies in Mathematics, 2(4): 389-399.

15. Kleene, S. (1967) Logiquemathématique. Amsterdam. North-Holland Publishing Company.

16. Klymchuk, S. (2010). Counterexamples in Cálculus. Mathematical Association of América. Resource Materials. United States of América.

17. Knuth, E. J. y Ko, Y. (2013). Validating Proofs and Counterexamples Across Contente Domains: Practices of Importance for Mathematics Majors. The Journal of Mathematical Behavior, 32, 20-35.

18. Komatsu, K. (2010). Counter-examples for Refinement of Conjectures and Proofs in Primary School Mathematics. The Journal of Mathematical Behavior, 29(1), 1-10.

19. Komatsu, K., Jones, K., Ikeda, T., y Narazaki, A. (2017). Proof validation and modification in secondary school geometry. The Journal of Mathematical Behavior. 47, 1-15.

20. Lakatos, I. (1976). Preuves et refutations: essaisur la logique de la decouvertemathématique. Herman, París.

21. Lee, K. (2016). Studets' proof schemes for mathematical proving and disprovong of propositions. The Journal Mathematical Behavior, 41, 26-44.

22. Locia, E. (2000). Les contre-exemplesdansl'enseignement des mathematiques. Tesis doctoral no publicada, Universidad Paul Sebatier. Touluse, Francia.

23. Lozano, M. D. (2015). Argumentaciónabductiva y prueba en problemas de geometríaanalíticautilizandogeogebra. TercerColoquio de Doctorado, Departamento de MatemáticaEducativa, Cinvestav. México.

24. Mitchell, T. (1996). On examples, counterexamples, and proof by example.

25. Morales, A. (2008). El papel que juega el contraejemplo en la construcción de las definiciones en matemáticas: El caso de la función convexa. Tesis inédita de Maestría. Universidad Autónoma de Guerrero, México.

26. Polya, G (1958). Les mathématiques el le raisonnement plausible. París. Gauthier-Villar.

27. Stylianou, D., Chae, N., \& Blanton, M. (2006). Students' proof schemes: a closer look at what characterizes students' proof conceptions en Alatorre, S., Cortina, J.L., Sáiz, M., \& Méndez, A. (Eds.). Proceedings of the TwentyEighth Annual Meeting of the North American Chapter of the International Group for the Psychology of Mathematics Education. Mérida, Mexico: Universidad PedagógicaNacional. 2, 54-60.

28. Weber, K. (2009). How Syntactic Reasoners can Develop Undertstanding, Evaluate Conjetures, and Generate Counterexamples in Advanced Mathematics. The Journal of Mathematical Behavior, 28, 200-208.

29. Zazkis, R. y Chernoff, E. (2008). ¿What Makes a Counterexample Exemplary? Educational Studies in Mathematics, 68, 195-208. 